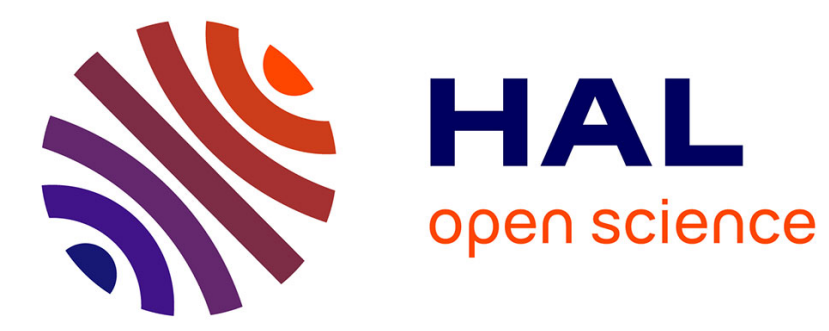

\title{
Rational Lyapunov functions and stable algebraic limit cycles
}

\author{
Emmanuel Moulay
}

\section{To cite this version:}

Emmanuel Moulay. Rational Lyapunov functions and stable algebraic limit cycles. IEEE Transactions on Automatic Control, 2014, 59 (4), pp.1077-1081. 10.1109/TAC.2013.2283757 . hal-01313557

\section{HAL Id: hal-01313557 \\ https://hal.science/hal-01313557}

Submitted on 23 Dec 2020

HAL is a multi-disciplinary open access archive for the deposit and dissemination of scientific research documents, whether they are published or not. The documents may come from teaching and research institutions in France or abroad, or from public or private research centers.
L'archive ouverte pluridisciplinaire HAL, est destinée au dépôt et à la diffusion de documents scientifiques de niveau recherche, publiés ou non, émanant des établissements d'enseignement et de recherche français ou étrangers, des laboratoires publics ou privés. 


\title{
Rational Lyapunov functions and stable algebraic limit cycles
}

\author{
Emmanuel Moulay
}

\begin{abstract}
The main goal of this article is to show that the class of systems described by a planar differential equation having a rational proper Lyapunov function has asymptotically stable sets which are either locally asymptotically stable equilibrium points, stable algebraic limit cycles or asymptotically stable algebraic graphics. The use of the Zubov equation is then an adapted tool to investigate the study of an upper bound on the number of stable limit cycles and asymptotically stable graphics and their relative positions for this class of systems.
\end{abstract}

Index Terms-Rational Lyapunov functions, algebraic limit cycles, Zubov equation, planar differential equations.

\section{INTRODUCTION}

The main idea of this article is to show that it is possible to investigate the stable limit cycles of a planar analytic system by using rational proper Lyapunov functions. We use the Zubov equation in this article rather than the Lyapunov equation. Both are partial differential equations, but the Zubov equation has a finite value at the boundary of the domain of attraction contrary to the Lyapunov equation. The Zubov equation which leads to the knowledge of the domain of attraction is an adapted tool to investigate the problem of the relative positions between the connected components of the domain of attraction of equilibrium points, limit cycles and graphics, even numerically. It is possible to study the existence of an upper bound for the number of stable limit cycles and asymptotically stable graphics for a special class of planar analytic ordinary differential equations which admits a rational proper Lyapunov function for each asymptotically stable set. In this case, the stable limit cycles but also the graphics are algebraic curves. The properness is essential to investigate the domain of attraction. In a very special case, it is also possible to have an upper bound for the number of limit cycles.

Let us recall that the Zubov theorem was given by Vladimir Ivanovich Zubov in the 1950s and appears in [1, Theorem 19]. This theorem gives a necessary and sufficient condition that characterizes the domain of attraction of an asymptotically stable set in terms of a nonlinear partial differential equation known as the Zubov equation. Notice that the topic of this article is related to Hilbert's sixteenth problem which is stated as follows:

- an investigation of the relative positions of the branches of real algebraic curves of degree $n$;

- the determination of an upper bound for the number of limit cycles $H(n)$ for planar ordinary differential

E. Moulay is with Xlim (UMR-CNRS 7252), Département SIC, Université de Poitiers, 11 bd Marie et Pierre Curie, BP 30179, 86962 Futuroscope Chasseneuil Cedex, France (e-mail: emmanuel.moulay@univ-poitiers.fr) equations of real polynomial vector fields of degree $n$ and an investigation of their relative positions.

This problem is unsolved and a survey about its history can be found in [2]. The stable limit cycles are one of the three kinds of limit cycles.

The article is organized as follows. After some notations and definitions given in Section II, a modified smooth version of the Zubov theorem for asymptotically stable sets is given in Section III. The Section IV is dedicated to the study of the class of systems which admits a polynomial proper Lyapunov function for each asymptotically stable set. It shows that this class is not suited for the study of the stable limit cycles. The right class is the one of systems which admits a rational proper Lyapunov function for each asymptotically stable set and its study is addressed in Section V. Finally a conclusion is given in Section VI.

\section{NotATiOnS AND DEFINITIONS}

For $x, y \in \mathbb{R}^{n}, x \cdot y$ denotes the scalar product between $x$ and $y$. A smooth function is a $C^{\infty}$ function.

Throughout this article, we consider a continuous, timeinvariant flow $\varphi: \mathbb{R} \times \mathbb{R}^{n} \rightarrow \mathbb{R}^{n}$. The flow $\varphi$ satisfies $\varphi(0, x)=x$ and $\varphi(t, \varphi(s, x))=\varphi(t+s, x)$ for all $t, s \in \mathbb{R}$ and $x \in \mathbb{R}^{n}$ (see for instance [3] and [4]).

Definition 1: A set $\mathcal{K} \subset \mathbb{R}^{n}$ is positively invariant if for all $x \in \mathcal{K}, \varphi(t, x) \in \mathcal{K}$ for all $t \in \mathbb{R}_{\geq 0}$.

We recall the definitions about Lyapunov stability.

Definition 2: A compact positively invariant set $\mathcal{K} \subset \mathbb{R}^{n}$ is asymptotically stable if the following two conditions hold:

1) $\mathcal{K}$ is stable: i.e. for every open neighborhood $\mathcal{V} \subseteq \mathbb{R}^{n}$ of $\mathcal{K}$, there exists an open neighborhood $\mathcal{U} \subseteq \mathbb{R}^{n}$ of $\mathcal{K}$ such that $\varphi(t, \mathcal{U}) \subseteq \mathcal{V}$ for every $t \geq 0$.

2) $\mathcal{K}$ is attractive: i.e. there exists an open neighborhood $\mathcal{W} \subseteq \mathbb{R}^{n}$ of $\mathcal{K}$ such that, for every $x \in \mathcal{W}$ and every open neighborhood $\mathcal{U} \subseteq \mathbb{R}^{n}$ of $\mathcal{K}$, there exists $T \geq 0$ such that $\varphi(t, x) \in \mathcal{U}$ for all $t>T$.

The domain of attraction of an asymptotically stable set $\mathcal{K} \subseteq \mathbb{R}^{n}$ is the set $\mathcal{A}$ of points $x$ such that, for every open neighborhood $\mathcal{U}$ of $\mathcal{K}$, there exists $T>0$ such that $\varphi(t, x) \in \mathcal{U}$ for all $t \geq T$.

$\mathcal{K} \subset \mathbb{R}^{n}$ is unstable if it is not stable.

The domain of attraction $\mathcal{A}$ of an asymptotically stable set $\mathcal{K}$ is an open invariant set containing a neighborhood of $\mathcal{K}[1$, Theorem 4].

Let $f: \mathbb{R}^{n} \rightarrow \mathbb{R}^{n}$ be a $C^{k}$ function with $k \geq 1$ and consider the system

$$
\dot{x}=f(x)
$$


where $x \in \mathbb{R}^{n}$. We assume that system (1) admits a global flow $\varphi(t, x)$ defined for all $t \in \mathbb{R}, x \in \mathbb{R}^{n}$ and given by the solutions satisfying $\varphi(0, x)=x$.

Let us define the notion of equilibrium point and periodic orbit.

Definition 3: Let $x_{0} \in \mathbb{R}^{n}, x_{0}$ is an equilibrium point of system (1) if $f\left(x_{0}\right)=0$. A solution $\varphi\left(t, x_{0}\right)$ of (1) is periodic if there exists a finite time $T>0$ such that $\varphi\left(t+T, x_{0}\right)=$ $\varphi\left(t, x_{0}\right)$ for all $t \geq 0$. A set $\mathcal{O} \subset \mathbb{R}^{n}$, not reduced to an equilibrium point, is a periodic orbit of (1) if

$$
\mathcal{O}=\left\{x \in \mathbb{R}^{n}: x=\varphi\left(t, x_{0}\right), 0 \leq t \leq T\right\}
$$

for some periodic solution $\varphi\left(t, x_{0}\right)$ of (1).

Even if it is possible to define a notion of stability, called orbital stability in [5, Section 4.2], for a periodic orbit in $\mathbb{R}^{n}$, we restrict the study to $\mathbb{R}^{2}$ with the more suited notion of limit cycle. Let us recall some definitions about limit cycles and their stability given in [6, Section 3.3].

Definition 4: Let $x \in \mathbb{R}^{n}$. The $\omega$-limit set of a trajectory $\varphi(., x)$ of (1) is defined by

$$
\omega(x):=\bigcap_{t \geq 0} \overline{\{\varphi(s, x): s \geq t\}}
$$

and the $\alpha$-limit set of a trajectory $\varphi(., x)$ of (1) is defined by

$$
\alpha(x):=\bigcap_{t \leq 0} \overline{\{\varphi(s, x): s \leq t\}} .
$$

A periodic orbit $\Gamma$ on $\mathbb{R}^{2}$ is called a limit cycle if it is the $\alpha$-limit set or $\omega$-limit set of some trajectory of (1) outside $\Gamma$ : i.e. $\Gamma$ is the set of accumulation points of either some forward or backward trajectory.

If $\Gamma$ is the $\omega$-limit set of every trajectory in some neighborhood of $\Gamma$ then $\Gamma$ is called stable. If $\Gamma$ is the $\alpha$-limit set of every trajectory in some neighborhood of $\Gamma$ then $\Gamma$ is called unstable. If $\Gamma$ is the $\alpha$-limit set of one trajectory other than $\Gamma$ and the $\omega$-limit set of one trajectory other than $\Gamma$ then $\Gamma$ is called semi-stable.

A limit cycle is a compact invariant set. A stable limit cycle is also called an asymptotically stable limit cycle to be in accordance with the asymptotic stability in the sense of Lyapunov in the Definition 2. It is worth noting that the unstability of a limit cycle is also in accordance with the unstability in the sense of Lyapunov [1, Definition 2].

Let us a give a more precise classification of semi-stable limit cycles given in [7]. The classical notions of interior and exterior are defined in [6, pp. 204-205].

Definition 5: A semi-stable limit cycle $\Gamma=\omega(x)$ on $\mathbb{R}^{2}$ with $x \notin \Gamma$ is

- semi-stable of type- 1 if $x$ is at the exterior of $\Gamma$.

- semi-stable of type-2 if $x$ is at the interior of $\Gamma$.

- $\infty$-semi-stable limit cycle if $\Gamma$ is semi-stable of type-2 and $\|\varphi(t, x)\| \underset{t \rightarrow+\infty}{\longrightarrow}+\infty$ if $x$ belongs to the exterior of $\Gamma$.

Let us give the definition of an algebraic closed curve and an algebraic limit cycle which are studied in [8, Section 7.2].

Definition 6: A closed curve is called an algebraic closed curve if it is a connected component of the zero set of some real polynomial function. A limit cycle is an algebraic limit cycle if it is a limit cycle and an algebraic closed curve.

Let us give the definition of a graphic. For more details and some examples, the reader may refer to the Definition 1 in $[6$, Section 3.7].

Definition 7: A graphic in $\mathbb{R}^{2}$ is a finite union of homoclinic and heteroclinic orbits compatibly oriented and connecting a finite number of equilibrium points in a cycle chain $x_{1} \rightarrow x_{2} \rightarrow \cdots \rightarrow x_{k} \rightarrow x_{1}$. It consists of equilibrium points and orbits having these equilibrium points as their $\alpha-$ and $\omega$-limit sets such that the orbits are compatibly oriented.

If the equilibrium points in the cycle chain are consecutively repeated, one obtains homoclinic orbits which are also called petals. A graphic is a compact invariant set which is not a periodic orbit.

Let us recall the definition of a Lyapunov function.

Definition 8: Let $\mathcal{K} \subset \mathbb{R}^{n}$ be a compact positively invariant set of system (1) and $\mathcal{A} \subset \mathbb{R}^{n}$ an open positively invariant set of system (1) containing a neighborhood of $\mathcal{K}$. A continuously differentiable function $W: \mathcal{A} \rightarrow \mathbb{R}_{\geq 0}$ such that

- $W(\mathcal{K})=0$

- $W(x)>0$ for all $x \in \mathcal{A} \backslash \mathcal{K}$;

is a Lyapunov function for system (1) if the Lyapunov equation

$$
\nabla W(x) \cdot f(x)=-h(x)
$$

holds for all $x \in \mathcal{A}$ with $h: \mathcal{A} \rightarrow \mathbb{R}_{>0}$ a positive function such that $h(\mathcal{K})=0$ and $h(x)>0$ for all $x \in \mathcal{A} \backslash \mathcal{K}$. Moreover if $W(x) \rightarrow \infty$ when $\|x\|$ approaches $\partial \mathcal{A}$ then the Lyapunov function is said to be proper.

A converse Lyapunov theorem refers to a necessary condition for asymptotic stability of a compact positively invariant set involving a Lyapunov function. The reader may refer to Theorem 2.2 in [4, page 66] for a converse Lyapunov theorem involving a continuous Lyapunov function and [9], [10], [11] for a converse Lyapunov theorem involving smooth and/or proper Lyapunov function. Let us remark that in Theorem 2.2 in [4, page 66], the state space is only supposed to be a locally compact set.

\section{THE ZUBOV THEOREM FOR ASYMPTOTICALLY STABLE SETS}

We will use in the following the Zubov theorem for asymptotically stable sets with the Zubov equation rather than the Lyapunov theorem for asymptotically stable sets given in [4], [11] with the Lyapunov equation (5). The main reason is the fact that a proper Lyapunov function has an infinite value at the boundary $\partial \mathcal{A}$ of the domain of attraction which is a not a good feature for solving, even numerically, a partial differential Lyapunov equation (5) and for finding $\mathcal{A}$.

Let us give a smooth modified version of the original Zubov theorem given in [1, Theorem 19] for asymptotically stable sets. The following result first appears in [9, Theorem 2].

Theorem 9 (Zubov theorem): Let $\mathcal{K} \subset \mathbb{R}^{n}$ be a compact positively invariant set of system (1) and $\mathcal{A} \subset \mathbb{R}^{n}$ an open positively invariant set of system (1) containing a neighborhood of $\mathcal{K}$. $\mathcal{A}$ is the domain of attraction of the asymptotically stable 
set $\mathcal{K}$ if and only if there exist a smooth function $V: \mathcal{A} \rightarrow \mathbb{R}$ and a $C^{k}$ function $h: \mathcal{A} \rightarrow \mathbb{R}$ such that:

- $V(\mathcal{K})=h(\mathcal{K})=0$;

- $-1<V(x)<0$ and $h(x)>0$ for all $x \in \mathcal{A} \backslash \mathcal{K}$;

- if $\mathcal{A}$ is bounded then $\lim _{x \rightarrow \partial \mathcal{A}} V(x)=-1$, otherwise $\lim _{\|x\| \rightarrow+\infty} V(x)=-1$;

- the Zubov equation

$$
\nabla V(x) \cdot f(x)=h(x)(1+V(x))
$$

holds for all $x \in \mathcal{A}$.

Moreover, we have $V^{-1}((-1,0])=\mathcal{A}$ and $V^{-1}(0)=\mathcal{K}$.

Proof: Sufficiency. Suppose that there exists a smooth function $V: \mathcal{A} \rightarrow \mathbb{R}$ and a $C^{k}$ function $h: \mathcal{A} \rightarrow \mathbb{R}$ such that the conditions of the theorem are satisfied. Then the smooth function

$$
W=-\ln (1+V)
$$

is a proper Lyapunov function for system (1). By using the Lyapunov theorem for asymptotically stable sets given in [4, page 66], we have that $\mathcal{K}$ is asymptotically stable. Moreover, by using [9, Theorem 1] and [12, Theorem 1] on each connected component of $\mathcal{A}$, we obtain that $\mathcal{A}$ is the domain of attraction.

Necessity. If $\mathcal{K}$ is asymptotically stable with a domain of attraction $\mathcal{A}$ then there exists a converse Lyapunov theorem involving a smooth proper Lyapunov function $W: \mathcal{A} \rightarrow \mathbb{R}_{\geq 0}$ [9], [10], [11]. It is obvious to check that the smooth function

$$
V=e^{-W}-1
$$

satisfies the Zubov equation (6) with

$$
h(x)=-\nabla W(x) \cdot f(x) .
$$

Moreover, the couple $(V, h)$ satisfies all the conditions of the theorem.

Remark 10: The original Zubov theorem [1, Theorem 19] involves continuous only functions $V$ and $h$. However $h$ is supposed to be continuous and positive over $\mathbb{R}^{n} \backslash \mathcal{K}$ which is a severe condition. As explained in [9, Theorem 2] and [12, Corollary 3.1], this condition can be replaced by a mild condition of regularity and positiveness of $h$ over $\mathcal{A} \backslash \mathcal{K}$ because the properness is enough to ensure that $\mathcal{A}$ is the domain of attraction. This fact has also been mentioned in [13, Theorem 2.11]. The Theorem 9 is also true if $f$ is only Lipschitz continuous on $\mathcal{A}$ and in this case $h$ is also Lipschitz continuous on $\mathcal{A}$.

Let us give an example of an asymptotically stable limit cycle by using the Zubov equation (6).

Example 11: Let $\mu>0$ and consider the system

$$
\left\{\begin{array}{l}
\dot{x_{1}}=x_{2}+\mu x_{1}\left(1-x_{1}^{2}-x_{2}^{2}\right) \\
\dot{x_{2}}=-x_{1}+\mu x_{2}\left(1-x_{1}^{2}-x_{2}^{2}\right)
\end{array}\right.
$$

Let

$$
h\left(x_{1}, x_{2}\right)=\frac{2 \mu\left(1-x_{1}^{2}-x_{2}^{2}\right)^{2}\left(1+x_{1}^{2}+x_{2}^{2}\right)}{x_{1}^{2}+x_{2}^{2}}
$$

and the Zubov equation associated with system (10) is given by

$$
\begin{aligned}
& \frac{\partial V}{\partial x_{1}}\left(x_{2}+\mu x_{1}\left(1-x_{1}^{2}-x_{2}^{2}\right)\right)+ \\
& \frac{\partial V}{\partial x_{2}}\left(-x_{1}+\mu x_{2}\left(1-x_{1}^{2}-x_{2}^{2}\right)\right)=h\left(x_{1}, x_{2}\right)(1+V) .
\end{aligned}
$$

A solution of the Zubov equation is given by

$$
V\left(x_{1}, x_{2}\right)=e^{-\frac{\left(1-x_{1}^{2}-x_{2}^{2}\right)^{2}}{x_{1}^{2}+x_{2}^{2}}}-1
$$

where $\mathcal{K}=\left\{\left(x_{1}, x_{2}\right) \in \mathbb{R}^{2}: x_{1}^{2}+x_{2}^{2}=1\right\}$ and the domain of attraction is given by $\mathcal{A}=\mathbb{R}^{2} \backslash\{(0,0)\}$. Thus, $\left\{\left(x_{1}, x_{2}\right) \in \mathbb{R}^{2}: x_{1}^{2}+x_{2}^{2}=1\right\}$ is an asymptotically stable limit cycle.

It is worth noting that, in control theory, the Zubov theorem generally refers to [1, Theorem 22] dedicated to the case of an equilibrium point, i.e. $\mathcal{K}=\{0\}$. A proof of this result is also given in [12, Theorem 3], [14, Theorem 34.1] and discussed in [13, Section 2.2.7]. A survey concerning the solution of the Zubov equation in the case of an equilibrium point can be found in [15, Section II.A] and some recent extensions are given in [16]. Some examples using the Zubov equation for the study of an equilibrium point are given in [1].

\section{POLYNOMIAL PROPER LYAPUNOV FUNCTIONS}

Let us consider a planar system

$$
\left\{\begin{array}{c}
\dot{x}_{1}=f_{1}\left(x_{1}, x_{2}\right) \\
\dot{x}_{2}=f_{2}\left(x_{1}, x_{2}\right)
\end{array}\right.
$$

where $f_{1}, f_{2}$ are analytic functions.

Let us denote by $\mathcal{P}$ the class of systems (14) admitting a polynomial proper Lyapunov function $W\left(x_{1}, x_{2}\right)$ for each asymptotically stable set. Such a class of systems is widely studied in the case of an asymptotically stable equilibrium point, see for instance [17], [18]. However, we prove in the following that this class of systems is not suited for studying stable limit cycles.

Theorem 12: Let $S \in \mathcal{P}$ then $S$ has no stable limit cycles.

Proof: Suppose that $S$ has a stable limit cycle $\Gamma$. As $S \in \mathcal{P}$, there exists a polynomial proper Lyapunov function $W\left(x_{1}, x_{2}\right)$ for each asymptotically stable set $\mathcal{K}$ which is characterized by a Zubov equation (6) whose solution is of the form $V\left(x_{1}, x_{2}\right)=e^{-W\left(x_{1}, x_{2}\right)}-1$. Let us consider the set $\mathcal{K}=\Gamma$. We have $\mathcal{K}=V^{-1}(0)=W^{-1}(0)$. Due to the Zubov theorem $9, W\left(x_{1}, x_{2}\right)$ must be singular on $\partial \mathcal{A}$, i.e. $\lim _{\left(x_{1}, x_{2}\right) \rightarrow \partial \mathcal{A}} W\left(x_{1}, x_{2}\right)=+\infty$. In particular, $W\left(x_{1}, x_{2}\right)$ must be singular on a bounded set included in $\Gamma$, which is not possible with a polynomial function.

The polynomial proper Lyapunov functions are not suited to the study of limit cycles, but rather to the study of asymptotically stable equilibrium points. A non proper Lyapunov function still remains as a possible solution to prove the asymptotic stability. For instance, the polynomial Lyapunov function

$$
W(x, y)=\frac{\left(x^{2}+y^{2}\right)^{2}}{4}-\frac{x^{2}+y^{2}}{2}+\frac{1}{4}
$$

can be used for the stable limit cycle of system (10). However, if the Lyapunov function $W$ is not proper then it is not possible to ensure that the domain of definition of $W$ coincides with 
the domain of attraction (see [9], [12]). So, the properness is a key point to address the study of the asymptotic stability involving the domain of attraction.

The polynomial Lyapunov functions can also be excluded for some systems of the form (14) having a globally asymptotically stable equilibrium point, see for instance [19].

The following example shows that a Zubov equation involving a natural function $h$ for a locally asymptotically stable equilibrium point embedded in a limit cycle leads to a rational proper Lyapunov function. Even if it does not exclude the existence of other types of proper Lyapunov functions, it also indicates that polynomial proper Lyapunov functions are too restrictive for the study of limit cycles.

Example 13: Consider system (10) of the example 11 with $\mu<0$. Let $0 \leq x_{1}^{2}+x_{2}^{2}<1$ and consider the function

$$
h\left(x_{1}, x_{2}\right)=-2 \mu \frac{x_{1}^{2}+x_{2}^{2}}{1-x_{1}^{2}-x_{2}^{2}}
$$

and the Zubov equation associated to system (10)

$$
\begin{aligned}
& \frac{\partial V}{\partial x_{1}}\left(x_{2}+\mu x_{1}\left(1-x_{1}^{2}-x_{2}^{2}\right)\right)+ \\
& \frac{\partial V}{\partial x_{2}}\left(-x_{1}+\mu x_{2}\left(1-x_{1}^{2}-x_{2}^{2}\right)\right)=h\left(x_{1}, x_{2}\right)(1+V) .
\end{aligned}
$$

A solution of the Zubov equation is given by

$$
V\left(x_{1}, x_{2}\right)=e^{\frac{x_{1}^{2}+x_{2}^{2}}{x_{1}^{2}+x_{2}^{2}-1}}-1
$$

where $\mathcal{K}=\{(0,0)\}$ and the domain of attraction is given by the set $\mathcal{A}=\left\{\left(x_{1}, x_{2}\right) \in \mathbb{R}^{2}: x_{1}^{2}+x_{2}^{2}<1\right\}$. Thus, $\{(0,0)\}$ is an asymptotically stable equilibrium point and it is possible to show that $\partial \mathcal{A}=\left\{\left(x_{1}, x_{2}\right) \in \mathbb{R}^{2}: x_{1}^{2}+x_{2}^{2}=1\right\}$ is an unstable limit cycle (see [7]).

It is worth noting that polynomial proper Lyapunov functions could be used with semi-stable limit cycles, for instance for a system having only embedded semi-stable limit cycles of type-1 with an asymptotically stable equilibrium point embedded in the smaller semi-stable limit cycle.

\section{RATIONAL PROPER LYAPUNOV FUNCTIONS}

Let us denote by $\mathcal{F}_{\nu, \delta}$ the class of systems of the form (14) admitting a rational proper Lyapunov function $W\left(x_{1}, x_{2}\right)=$ $\frac{N\left(x_{1}, x_{2}\right)}{D\left(x_{1}, x_{2}\right)}$ in which the degree of $N\left(x_{1}, x_{2}\right)$ is lower than $\nu>0$ and the degree of $D\left(x_{1}, x_{2}\right)$ is lower than $\delta>0$ for each asymptotically stable set. This class $\mathcal{F}_{\nu, \delta}$ is more interesting than $\mathcal{P}$ because it allows the study of stable limit cycles. The number of stable limit cycles and asymptotically stable graphics of a system in $\mathcal{F}_{\nu, \delta}$ is denoted by $H_{s}(\nu, \delta)$.

Theorem 14: Let $S \in \mathcal{F}_{\nu, \delta}$ then all asymptotically stable sets are either:

- locally asymptotically stable equilibrium points;

- stable algebraic limit cycles;

- asymptotically stable algebraic graphics;

and $H_{s}(\nu, \delta) \leq \frac{\nu}{2}$. For all stable algebraic limit cycles $\Gamma=$ $\omega(x)$ with $x=\left(x_{1}, x_{2}\right) \notin \Gamma$, all bounded $\alpha(x)$ are planar algebraic closed curves and their number is lower than $\frac{\delta}{2}$.

Proof: As $S \in \mathcal{F}_{\nu, \delta}$, there exists a rational proper Lyapunov function $W\left(x_{1}, x_{2}\right)=\frac{N\left(x_{1}, x_{2}\right)}{D\left(x_{1}, x_{2}\right)}$ whose degree of $N\left(x_{1}, x_{2}\right)$ is lower than $\nu$ and whose degree of $D\left(x_{1}, x_{2}\right)$ is lower than $\delta$ for each asymptotically stable set which is characterized by a Zubov equation (6) whose solution is of the form $V\left(x_{1}, x_{2}\right)=e^{-W\left(x_{1}, x_{2}\right)}-1$ (see the Theorem 9 ). Let us consider the set $\mathcal{K}$ defined by the union of all asymptotically stable equilibrium points, stable limit cycles and asymptotically stable graphics. Due to the PoincaréBendixson theorem for analytic systems [6, Page 245], the only possible asymptotically stable sets are asymptotically stable equilibrium points, stable limit cycles and asymptotically stable graphics. Moreover, the asymptotically stable equilibrium points cannot be globally asymptotically stable due to the Zubov theorem (9) and the fact that $\delta>0$. There exists $V\left(x_{1}, x_{2}\right)=e^{-W\left(x_{1}, x_{2}\right)}-1$ with $W\left(x_{1}, x_{2}\right)=\frac{N\left(x_{1}, x_{2}\right)}{D\left(x_{1}, x_{2}\right)}$ such that $\mathcal{K}=V^{-1}(0)=N^{-1}(0)$. So, the stable limit cycles and the asymptotically stable graphics are algebraic curves and factors of the polynomial $N\left(x_{1}, x_{2}\right)$. As a planar algebraic closed curve is defined by an equation of degree larger than 2 (see for instance [20]), the number of stable limit cycles and asymptotically stable graphics is lower than $\frac{\nu}{2}$.

For a stable limit cycle $\Gamma=\omega(x)$ with $x=\left(x_{1}, x_{2}\right) \notin \Gamma$, the set $\alpha(x)$ is included in $\partial \mathcal{A}$ if it is bounded. Moreover, we have $\partial \mathcal{A}=V^{-1}(-1)$. The only bounded sets in $V^{-1}(-1)$ are given by $D^{-1}(0)$. Due to the Poincaré-Bendixson theorem for analytic systems, $\alpha(x)$ is a closed curve. If $\alpha(x) \subset D^{-1}(0)$ then it is a planar algebraic closed curve and a factor of the polynomial $D\left(x_{1}, x_{2}\right)$. As a planar algebraic closed curve is defined by an equation of degree larger than 2 , the number of bounded $\alpha(x)$ is lower than $\frac{\delta}{2}$.

The domain of attraction $\mathcal{A}$ given by the solution of the Zubov equation (6) provides the relative positions between the connected components of the domain of attraction of locally asymptotically stable equilibrium points, the stable algebraic limit cycles and the asymptotically stable algebraic graphics. Even if it is possible to know the existence of a rational proper Lyapunov function $W$, it is in general very difficult to have an explicit expression for $W$ whereas it is possible to compute, even numerically, a solution for $V$ and $\mathcal{A}$ with the Zubov equation (6).

Example 15: It is worth noting that a graphic can be an asymptotically stable set. Consider for instance the system in polar coordinates

$$
\left\{\begin{array}{l}
\dot{r}=r(r-1) \\
\dot{\theta}=\cos ^{2} \theta
\end{array}\right.
$$

It has a homoclinic orbit, the unit sphere, which is an asymptotically stable graphic.

Let us denote by $\mathcal{F}_{\nu, \delta}^{\infty}$ the subclass of systems in $\mathcal{F}_{\nu, \delta}$ having no graphic nor $\infty$-semi-stable limit cycle. The number of limit cycles of a system in $\mathcal{F}_{\nu, \delta}^{\infty}$ is denoted by $H^{\infty}(\nu, \delta)$.

Corollary 16: Let $S \in \mathcal{F}_{\nu, \delta}^{\infty}$ then all limit cycles are algebraic limit cycles and $H^{\infty}(\nu, \delta) \leq \frac{\delta}{2}+\frac{\nu}{2}$.

Proof: As $S \in \mathcal{F}_{\nu, \delta}^{\infty}$, there exists a rational proper Lyapunov function $W\left(x_{1}, x_{2}\right)=\frac{N\left(x_{1}, x_{2}\right)}{D\left(x_{1}, x_{2}\right)}$ whose degree of $N\left(x_{1}, x_{2}\right)$ is lower than $\nu$ and whose degree of $D\left(x_{1}, x_{2}\right)$ is lower than $\delta$ for each asymptotically stable set which is characterized by a Zubov equation (6) whose solution is of the form $V\left(x_{1}, x_{2}\right)=e^{-W\left(x_{1}, x_{2}\right)}-1$. Let us consider the set 
$\mathcal{K}$ defined by the union of all asymptotically stable equilibrium points, stable limit cycles and and semi-stable limit cycles. The semi-stable limit cycles can be considered in $\mathcal{K}$. Indeed, due to the Poincaré-Bendixson theorem for analytic systems [6, Page 245] and the assumptions, there are two possibilities for a semi-stable limit cycle:

- either its unstable $\alpha$-limit side converges toward an asymptotically stable equilibrium point, or a stable limit cycle, or a semi-stable limit cycle which are all in $\mathcal{K}$. It means that every solution in a neighborhood of the semi-stable limit cycle converges asymptotically toward $\mathcal{K}$.

- either its unstable $\alpha$-limit side diverges at infinity.

Due to the assumption that $S$ has any $\infty$-semi-stable limit cycle, the second case is excluded. There exists $V\left(x_{1}, x_{2}\right)=$ $e^{-W\left(x_{1}, x_{2}\right)}-1$ with $W\left(x_{1}, x_{2}\right)=\frac{N\left(x_{1}, x_{2}\right)}{D\left(x_{1}, x_{2}\right)}$ such that $\mathcal{K}=$ $V^{-1}(0)=N^{-1}(0)$. So, the stable and semi-stable limit sets are algebraic limit cycles characterized by the polynomial $N\left(x_{1}, x_{2}\right)$. As a planar algebraic closed curve is defined by an equation of degree larger than 2 , the number of stable and semi-stable limit cycles is lower than $\frac{\delta}{2}$.

Due to the Poincaré-Bendixson theorem for analytic systems [6, Page 245] and the assumptions, the unstable limit cycles belong to the set $\partial \mathcal{A}$. Indeed, let $x$ belongs to the interior of an unstable limit cycle $\Gamma=\alpha(x)$ then $\omega(x)$ is either an equilibrium point, either a limit cycle. So $\omega(x)$ is an asymptotically stable point or a stable limit cycle or a semistable limit cycle and thus we have $\Gamma \subset \partial \mathcal{A}$. Moreover, we have $\partial \mathcal{A}=V^{-1}(-1)$. As the only bounded sets in $V^{-1}(-1)$ are given by $D^{-1}(0)$, the unstable limit cycles are algebraic limit cycles and factors of the polynomial $D\left(x_{1}, x_{2}\right)$. A planar algebraic closed curve being defined by an equation of degree larger than 2, the number of unstable limit cycles is lower than $\frac{\nu}{2}$.

As the limit cycles are stable, semi-stable or unstable, the result follows.

At the best of our knowledge, there is no result in the literature concerning a converse Lyapunov result involving rational proper Lyapunov functions. However, such a result should be interesting to investigate the stable algebraic limit cycles and the asymptotically stable algebraic graphics. It seems that there is an intriguing link between the class $\mathcal{F}_{\nu, \delta}$ and the algebraic limit cycles.

An interesting case concerns the exponential stability. It has been shown in [18] that if we consider exponential stability only of an equilibrium point on a bounded domain rather than asymptotic stability, then it is possible to find a polynomial Lyapunov function $W(x)$ whose degree is lower than an upper bound which depends on the Lipschitz bound of the system and also the rate of exponential convergence. We may wonder if this result can be extended to the neighborhood of a limit cycle. Indeed, under some assumptions, all solutions starting on a neighborhood of a limit cycle decay exponentially toward the limit cycle (see [21]).

\section{CONCLUSION}

This article is a first step highlighting a link between the rational proper Lyapunov functions and the stable algebraic limit cycles for a planar system. However, further studies of the class $\mathcal{F}_{\nu, \delta}$ are necessary in order to determine and to classify the systems which are in the scope of this article.

\section{REFERENCES}

[1] V. I. Zubov, Methods of A.M. Lyapunov and their Application. Noordhoff The Netherlands, 1964.

[2] Y. Ilyashenko, "Centennial history of Hilbert's 16th problem," Bulletin of the American Mathematical Society, vol. 39, no. 3, pp. 301-354, 2002.

[3] N. P. Bhatia and O. Hajek, Local semi-dynamical systems. Lecture Notes in Mathematics, vol. 90, Springer, 1969.

[4] N. P. Bhatia and G. P. Szegő, Stability Theory of Dynamical Systems. Classics in Mathematics, Springer, 2002.

[5] A. Medio and M. Lines, Nonlinear dynamics: A primer. Cambridge University Press, 2001.

[6] L. Perko, Differential equations and dynamical systems, ser. Texts in Applied Mathematics. Springer, 2000, vol. 7.

[7] A. Ghaffari, M. Tomizuka, and R. Soltan, "The stability of limit cycles in nonlinear systems," Nonlinear Dynamics, vol. 56, no. 3, pp. 269-275, 2009.

[8] F. Dumortier, J. Llibre, and J. Artés, Qualitative theory of planar differential systems, ser. Universitext. Springer, 2006.

[9] L. T. Grujić, "Solutions to Lyapunov stability problems of sets: nonlinear systems with differentiable motions," International Journal of Mathematics and Mathematical Sciences, vol. 17, no. 1, pp. 103-112, 1994.

[10] Y. Lin, E. D. Sontag, and Y. Wang, "A smooth converse Lyapunov theorem for robust stability," SIAM Journal on Control and Optimization, vol. 34, no. 1, pp. 124-160, 1996.

[11] F. Wilson, "Smoothing derivatives of functions and applications," Transactions of the American Mathematical Society, pp. 413-428, 1969.

[12] A. Vannelli and M. Vidyasagar, "Maximal Lyapunov functions and domains of attraction for autonomous nonlinear systems," Automatica, vol. 21, no. 1, pp. 69-80, 1985.

[13] A. Bacciotti and L. Rosier, Liapunov functions and stability in control theory. Springer, 2005.

[14] W. Hahn, Stability of motion. Springer-Verlag New York, 1967, vol. 138.

[15] R. Genesio, M. Tartaglia, and A. Vicino, "On the estimation of asymptotic stability regions: State of the art and new proposals," IEEE Transactions on Automatic Control, vol. 30, no. 8, pp. 747-755, 1985.

[16] F. Camilli, L. Grüne, and F. Wirth, "A generalization of Zubov's method to perturbed systems," SIAM Journal on Control and Optimization, vol. 40, no. 2, pp. 496-515, 2001.

[17] M. Peet, "Exponentially stable nonlinear systems have polynomial Lyapunov functions on bounded regions," IEEE Transactions on Automatic Control, vol. 54, no. 5, pp. 979-987, 2009.

[18] M. Peet and A. Papachristodoulou, "A converse sum of squares Lyapunov result with a degree bound," IEEE Transactions on Automatic Control, vol. 57, no. 9, pp. 2281-2293, 2012.

[19] A. A. Ahmadi, "Algebraic relaxations and hardness results in polynomial optimization and Lyapunov analysis," Ph.D. dissertation, Massachusetts Institute of Technology, 2011.

[20] G. Fischer, Plane algebraic curves, ser. Student Mathematical Library. American Mathematical Society, 2001, vol. 15.

[21] B. Aulbach, "Asymptotic stability regions via extensions of Zubov's method- II." Nonlinear Analysis: Theory, Methods \& Applications, vol. 7, no. 12, pp. 1441-1454, 1983. 